\title{
LOS FEMINISMOS EN EL SIGLO XXI. PLURALIDAD DE PENSAMIENTOS
}

\author{
Prudencia Gutiérrez Esteban \\ María Rosa Luengo González \\ Universidad de Extremadura
}

RESUMEN: En este artículo se realiza un breve recorrido por las corrientes feministas y se aporta nuestra visión sobre los problemas que están abordando las feministas en el momento actual, reflexionando acerca de cuáles son los retos planteados y qué elementos están incidiendo en la evolución de los mismos.

Asimismo, se incide en tres de las grandes líneas de los feminismos: el feminismo trans, el feminismo y la inmigración y el ciberfeminismo.

Finalmente revisamos la investigación en estudios de género como elemento de reflexión y de generación de conocimiento científico que realiza propuestas de cambio social.

Palabras clave: Feminismos, teorías, pluralidad.

\section{THE FEMINISMS IN THE 21ST CENTURY. PLURALITY OF THOUGHTS}

ABSTRACT: In this paper are described the different feminist movements and brings our views on the issues that feminists are focusing nowadays, reflecting about what are the challenges and elements that are influencing on its evolution.

It is also studied the three main research areas of feminism: trans feminism, feminism and immigration and cyberfeminism.

We also review the research in gender studies as a reflection element and generation of scientific knowledge, making proposals for social change.

Keywords: Feminism, theory, plurality. 


\section{Introducción}

Para entender la evolución del movimiento feminista hasta nuestros días es necesario hacer un poco de historia y resumir los hitos más importantes por lo que éste ha ido pasando, así como recorrer los nuevos feminismos y los temas que están abordando en la actualidad.

Desde siempre han existido mujeres que de una forma u otra, a lo largo de la Historia, han denunciado y luchado por mejorar sus condiciones vitales, por lo que podemos asegurar junto con Ana de Miguel (1995), que el feminismo ha existido desde siempre, aunque los movimientos más o menos organizados que han protagonizado la lucha por los derechos de las mujeres tienen lugar a lo largo de los siglos XIX y XX. Principalmente, destacamos dos grandes momentos históricos: el primero, a finales del siglo XIX hasta mediados del siglo $\mathrm{XX}$, con las primeras sufragistas, que podemos denominar "Feminismo Moderno", y el segundo, a partir de de los años 60 hasta la actualidad, denominado "Feminismo Contemporáneo".

El Feminismo Moderno tiene sus raíces en los movimientos de mujeres que participaron en la Revolución Francesa (1789), donde se crearon los Clubs de Mujeres (Salonières), teniendo su continuación a la sombra de los grandes movimientos sociales que tuvieron lugar en el siglo XIX: el socialismo, el marxismo, el anarquismo y por supuesto, en el movimiento sufragista, así como en los feminismos contemporáneos y los de las últimas tendencias.

En esta primera época, las mujeres exponen sus ideas sobre el derecho natural, la justicia y la democracia, en conexión con las nuevas corrientes del individualismo liberal y de la Ilustración, dando lugar al feminismo liberal que se ocupa de ampliar los derechos legales, políticos y laborales de las mujeres de la clase media. Sus conquistas más importantes son las mejoras de la legislación sobre la propiedad en el matrimonio, el acceso a la educación y a las profesiones y mayores oportunidades de empleo y de participación de las mujeres en el gobierno y en la vida pública.

Las mujeres pertenecientes a este movimiento, entienden que el acceso a la educación es fundamental para la igualdad de derechos y oportunidades. Proporcionando una educación igual a ambos sexos se podría estimular y desarrollar el potencial de cada persona. Asimismo, creen que tanto las mujeres como los hombres deben gozar de igual libertad para decidir cuáles deben ser sus funciones políticas, sociales y educativas, y abolir todas aqueIlas tradiciones, leyes y actividades que impidan la igualdad de derechos y oportunidades.

El Feminismo Contemporáneo tuvo sin embargo unos principios más revolucionarios. En la década de los 60 en Estados Unidos nace el Women's Liberation Movement (WLM) que fundamentado en las teorías marxistas, entiende que la sociedad está compuesta por un sistema de sexos y clases sociales, lo que les lleva a estudiar el papel de las mujeres en las organizaciones políticas 
y a impulsar su propio movimiento de liberación. En esta época destaca el Feminismo radical: Reunía a grupos y posiciones teóricas de las feministas del momento. Se desarrolló durante los años 1967 y 1975 en E.E.U.U. fundamentalmente. Cabe destacar a Kate Miller (1971) con la obra Política Sexual y Sulamith Firestone (1970) con La dialéctica de la sexualidad.

A partir de la década 80, se produce una escisión importante como consecuencia de los diferentes modelos de interpretación y en ella surgen una gran diversidad de opciones. Este movimiento es denominado por algunas autoras Postfeminismo. Las diferentes corrientes que lo integran son:

a) Feminismo lesbiano, mantiene que la homosexualidad lesbiana no es sólo una cuestión de preferencia sexual o de derechos civiles, sino una forma de vida que combina lo personal con lo político. Fueron las primeras que denunciaron públicamente la gravedad de la violencia contra las mujeres y propusieron la creación de centros de acogida. Su influencia en la sociedad actual ha sido decisiva.

b) Feminismo psicoanalítico, estudia el modo en que afecta a la vida emocional y la sexualidad de las mujeres la opresión que sufren. Afirma que las raíces de esta opresión están ancladas en la psique y para liberarse es necesario llevar a cabo una revolución interior.

c) Feminismo postmodernista, Se centra en buscar los fundamentos que demuestren que la filosofía y las teorías del conocimiento no son unitarias.

d) Feminismo postestructural, analiza la actuación del patriarcado en todas sus manifestaciones: ideológicas, institucionales, organizativas y subjetivas. Sostiene que los distintos significados de "ser mujer" se modifican y cambian a consecuencia de las variaciones y cambios discursivos en la cultura y en la historia, y por lo tanto, hay que ofrecer a las mujeres un espacio discursivo propio en el que puedan exponer sus puntos de vista.

e) Feminismo de la diferencia, basado en la diferencia sexual para crear un programa de liberación de las mujeres que alcance su auténtica identidad. Una de sus portavoces más notables es la francesa Luce Irigaray, quien junto con Kristeva y Cixous pretendieron demostrar en los años 80, las contradicciones internas de los sistemas de pensamiento predominantes, así como reinterpretar la teoría y la práctica psicoanalítica freudiana, aunque es en Italia donde se han desarrollado los trabajos que han tenido mayor impacto. Entre otras, hay que destacar de manera especial, a Ana $M^{a}$ Piussi del colectivo Diótima de Milán, quien explica cómo se abre esta perspectiva feminista en lo que concierne a la educación, logrando instalarse en el nombre de "Pedagogía de la diferencia sexual". El feminismo de la diferencia hace una crítica al feminismo de la igualdad porque considera que éste equipara las 
mujeres a los varones, instalándose de nuevo dentro de la dinámica de la dominación masculina (Varela, 2005¹).

f) Feminismo institucional, sus inicios se remontan al feminismo internacional de entreguerras, tras el Informe Mundial sobre el Estatus de la Mujer, realizado por la Liga de Naciones. A partir de ahí la situación de las mujeres fue asumida por los organismos internacionales, hasta el punto de que en 1946 se crea al amparo de Naciones Unidas la Comisión sobre el Estatus de las Mujeres. Hasta entonces, el feminismo se había desarrollado alejado del poder. Es entonces cuando van teniendo lugar sucesivamente las distintas conferencias mundiales de la ONU sobre la Mujer en México (1975), Copenhague (1980), Nairobi (1985), Beijing (1995) y Nueva York (2000).

Tras la revisión de las principales aportaciones que los diferentes feminismos han realizado a la evolución del pensamiento actual, debemos reconocer las contribuciones de otros feminismos como las del feminismo alemán De Sotelo $\left(2005^{2}\right)$ y el feminismo sudamericano con teóricas tan relevantes como Marcela Lagarde e Iris Zavala, cuyas tesis han tenido un notable impacto en nuestras visiones feministas.

Igualmente, existen otros feminismos derivados de diferentes corrientes de pensamiento, como el feminismo cristiano, el marxista, el libertario, el humanista, el ecofeminismo (Vandana Shiva en India) Agra $\left(1998^{3}\right)$ y Puleo $\left(2002^{4}\right)$, el feminismo musulmán, el cyberfeminismo (Schiaffino ${ }^{5}$, 2005; De Miguel y Boix $^{6}, 2002$; Boix ${ }^{7}$, 2007), el feminismo dialógico (Puigvert ${ }^{8} 2001$ y Arrufat Gallardo, ${ }^{9}$ 2004), el integral, el cultural, el separatista, el filosófico, el feminismo académico y los lobbys o grupos de presión, etc., que complementan la teoría feminista y reflejan las preocupaciones de las mujeres.

1. Varela, N., Feminismo para principiantes. Barcelona, 2005.

2. Sotelo, E. (ed.), New Women of Spain. Münster, 2005.

3. Agra, M. J. (comp.), Ecología y Feminismo. Granada, 1998.

4. Puleo, A., "Feminismo y ecología. Un repaso a las diversas corrientes del ecofeminismo" [en línea]. El Ecologista, 31 (2002). <http://www.fyl.uva.es/ wceg/articulos/ElEcologista. pdf> [consulta: 19/2/2007, 21:00].

5. Schiaffino, I., "Cyberfeminismo. El movimiento feminista y las mujeres en la comunidad virtual y real". En Ruiz-Tagle, A. $M^{a}$. (coord.), Los estudios de las mujeres hacia el Espacio Común Europeo. Sevilla, 2005.

6. De Miguel, A. y Boix, M., Los géneros de la red: los ciberfeminismos [en línea]. <http:// www.mujeresenred.net/> [consulta: 22/02/2007, 13:04]. 2007.

7. Boix Boix, M., Hackeando el patriarcado [En línea] <http://www.mujeresenred.net> [consulta: 22/02/2007, 13:04]. 2007.

8. Puigvert, L., Las otras mujeres. Barcelona, 2001.

9. Arrufat Gallardo, M., "Las otras mujeres y la Pedagogía de la Autonomía de Freire". Revista Interuniversitaria de Formación del Profesorado, 18 (2004), pp. 45-58. 
No podemos finalizar esta revisión por el amplio espectro de movimientos feministas, sin citar el movimiento de mujeres jóvenes feministas. Wilson ${ }^{10}$ (2006) describe algunos de los movimientos de mujeres jóvenes y sus relaciones con el feminismo; entre ellos, están las activistas de la justicia social, las feministas "underground", las feministas profesionales y la nueva generación de feministas. Esta nueva generación pretende ofrecer nuevos análisis, partiendo del marco teórico y de las experiencias de las anteriores generaciones feministas, nuevos puntos de vista desde los que analizar las relaciones de poder, proponer estrategias y formas de organizarse en el movimiento, hasta encontrar un equilibrio entre el discurso y la práctica. Centran su acción en torno a tres temas claves para las jóvenes feministas, la estrategia y la acción, la división del poder y tensiones multigeneracionales y la sostenibilidad.

\section{Los nuevos feminismos}

Hasta aquí podemos decir, que nos hemos aproximado a los distintos feminismos, clasificados según su adscripción a la corriente política, teórica o de pensamiento y según las aportaciones a la mejora de la situación de las mujeres y por ende, de la sociedad a lo largo de la historia. Así el feminismo a partir de lo que algunas autoras han denominado, la Segunda Ola "(...) no se ha manifestado de forma monolítica y universal, sino muy diversificado en sus métodos de actuación, en sus prioridades, e incluso, en sus fundamentaciones teóricas previas, que hunden su raíces ya en el marxismo o en el psicoanálisis, en el estructuralismo o el pensamiento postmoderno" (De Torres, 2004 en Freedman, 2004, 9-10).

Por lo que para autoras como Jane Freedman ${ }^{11}$ (2004) y Shamillah Wilson (2006), a partir de los años setenta hay que hablar de Feminismos, y no de Feminismo como un solo movimiento o una sola corriente de pensamiento. Lo cual no quiere decir que entre las diferentes opciones haya enfrentamientos; sino que es más lo que compartimos que lo que nos hace diferentes.

"Por eso, tras la explosión de los años setenta, la reacción de los ochenta y la escisión de los feminismos de los últimos años, la propuesta de todo el feminismo continúa siendo muy simple: exige que las mujeres tengan libertad para definir por sí mismas su identidad, en lugar de que ésta sea definida, una y otra vez, por la cultura de la que forman parte y los hombres que los que conviven" Varela (2005: 191).

10. Wilson, S. Wilson, S., "Mujeres jóvenes y movimientos feministas: ¿Una nueva generación de feministas?". En Gil Gómez, A., II Congreso Estatal de la Fundación Isonomía para la Igualdad de Oportunidades (FIIO) sobre igualdad entre mujeres y hombres: "Mujeres jóvenes: ¿Los nuevos feminismos?" Castellón de la Plana, 2006, pp. 49.

11. Freedman, J., Feminismo, ¿Unidad o conflicto?. Madrid, 2004. 
Para la nueva generación de feministas se hace necesario conformar un grupo crítico de mujeres jóvenes comprometidas que puedan enfrentarse a los retos que existen para ellas en nuestro contexto actual, desde distintas perspectivas y maneras de actuar, pues entiende "(...) la importancia de construir alianzas con la gente que comparte sus puntos de vista sobre justicia social y que apoyan a las que no se identifican abiertamente como feministas pero que forman parte de otros movimientos" (Wilson, 2006).

Así, en los inicios del siglo XXI, los movimientos feministas siguen existiendo, aunque con profundas transformaciones. Siendo una de las alternativas posibles, la que propone Celia Amorós $^{12}$ (2006) quien nos habla de un canon feminista intercultural que incluya todos los feminismos y continúe desde esta unidad, trabajando por la igualdad. Unidad que como propone el feminismo dialógico, debe ser inclusiva, dinámica e igualadora (Puigvert, 2001).

Como consecuencia de esta gran diversidad de movimientos feministas, en la revisión de la literatura, encontramos distintas posibilidades a la hora de narrar cómo han ido teniendo lugar los acontecimientos en la lucha para alcanzar la igualdad por parte de las mujeres.

Una de ellas es la que nos ofrece Carmen Blanco ${ }^{13}$ (1997), quien aunque hace una revisión histórica, clasifica las distintas corrientes y momentos en feminismo originario, que contendría los primeros brotes feministas y los primeros textos a favor de la igualdad entre mujeres y hombres, el feminismo sufragista, incluyendo la eclosión en distintos países europeos y Estados Unidos y el período entreguerras y por último, el feminismo de liberación de la mujer, que repasa las distintas teorías y situaciones sociales que coexistieron con éstas. Mientras que autoras como Amelia Valcárcel ${ }^{14}$ (2005) o Mary Nash ${ }^{15}$ (2002) optan por los distintos períodos históricos para clarificar y hablar de los feminismos -el de la Primera, Segunda y Tercera Ola-, Ana de Miguel ${ }^{16}$ (1995) los clasifica en lo que podríamos denominar familias teórico-ideológicas, citadas anteriormente o Jane Freedman (2004) que adopta la decisión de describir de forma crítica, los debates en los que se ha visto envuelto el feminismo, aportando luz a las cuestiones planteadas anteriormente desde las distintas tendencias y sensibilidades.

De este modo, Freedman (2004) opina que resumir la evolución de los movimientos feministas en "olas", puede llevar a pensar que entre esas "olas"

12. Amorós, C., (dir.), 10 palabras clave sobre mujer. Pamplona, 1995.

13. Blanco, C., El contradiscurso de las mujeres. Historia del feminismo. Vigo, 1997.

14. Valcárcel, A., "La memoria colectiva y los retos del feminismo" [en línea]. En Valcárcel, A. y Romero, R., Los desafíos del feminismo ante el siglo XXI. Sevilla, Instituto Andaluz de la Mujer. Col. Hipatia, 2005, pp. 19-54.

15. Nash, M., "Las mujeres en el mundo contemporáneo". Aula-Historia Social, 9 (2002), pp. 14-35.

16. De Miguel, A., "Feminisimos". En Amorós, C. (dir.), 10 palabras clave en mujer. Navarra, 1995, pp. 217-255. 
o períodos, no hubo actividad feminista, cuando en realidad, lo que ocurrió fue que las "feministas fueron quizás menos visibles y sus voces se escucharon menos (...). Por eso, aunque sea cierto que los movimientos feministas han sido más activos y han reclutado a más miembros en determinados períodos históricos, sería quizá más exacto ver el feminismo no como surgiendo en oleadas, sino como un continuo de pensamiento y acción" (op. cit., 2004:19).

Por otro lado, aunque dice que la clasificación que se hace entre grupos separados o íntimamente vinculados, puede resultar útil para comprender la complejidad del fenómeno feminista, tampoco la acepta pues "(...) parece haber una tendencia a pasar por alto las diferencias y dar más importancia a los elementos comunes dentro de las distintas categorías del feminismo, y, por el otro, a hacer hincapié en las diferencias entre unas categorías y otras" (op. cit.: 20). Por ello, Freedman opta por relacionar los feminismos conforme a "Ios distintos asuntos y problemas hacia los cuales han dirigido su atención las feministas (...) explorando los diferentes análisis que diversas feministas han aportado en relación con asuntos relevantes para las mujeres" (op. cit.: 21). Los asuntos que más han preocupado a las feministas y los principales debates y planteamientos que abordan son la participación política y la ciudadanía de las mujeres, su situación económica y su relación con el mercado de trabajo, la sexualidad y la reproducción y por último, uno de los principales debates teóricos, la cuestión de la "igualdad" y "diferencia" para el feminismo.

Por tanto, ya en el siglo XXI tenemos que hablar de feminismos en plural pues no existe un solo enfoque y cada vez son más diversas las miradas que tenemos las mujeres de explicar su experiencia vital, de vivir sus espacios de relación y de entender la vida, el mundo y las maneras de explicarse. Es una realidad que se han ido ampliando las diferencias en los puntos de vista de los análisis y los temas prioritarios de estudio y de reivindicación también han ido cambiando.

Hay varios elementos importantes a tener en cuenta en estos cambios, son:

a) El cambio generacional que implica la incorporación al movimiento feminista de las nuevas generaciones de mujeres que se enfrentan a retos nuevos y que parten de conquistas realizadas por las mujeres de anteriores generaciones. Esto implica que muchos de los logros antes conseguidos ahora se vean como realidades de hecho y de derecho sin discusión posible y en consecuencia se plantean otros problemas que, al mismo tiempo las generaciones anteriores no tuvieron. Las mujeres jóvenes están viviendo en una sociedad que maneja las redes sociales y tiene para la comunicación todo tipo de dispositivos que manejan sin ningún problema pues ellas ya pertenecen a la generación digital. Ello no implica que no existan retos que deben ser explicados para evitar las desigualdades, las que producen la Ilamada brecha digital. Como consecuencia surge el ciberfeminismo del que después hablaremos. 
b) La coexistencia de grupos de mujeres de diferentes países como consecuencia de la emigración ha hecho imprescindible abordar los problemas con los que se ven impelidas a afrontar. Son grupos muy diversos con orígenes y culturas muy distantes y visiones del mundo enfrentadas en muchos casos con la cultura y la realidad social de los nuevos lugares en los que se han incorporado. Estas mujeres, en general, van a asumirlos trabajos peor remunerados y en muchos casos se dedicarán a tareas de cuidado de mayores, bebés y personas con discapacidad, para permitir la conciliación de la vida laboral de las otras mujeres, pero sin resolver los suyos propios. Para resolver sus problemas y buscar formas de reivindicación han surgido movimientos feministas de mujeres inmigrantes.

c) Las nuevas formas de entender las construcciones sexo-género. La realidad trans. La aceptación de la diversidad de sexos-géneros implica comprender y asumir que la relación dicotómica sexo biológico-género dual ya no se sostiene pues existen otras realidades que se representan en personas intersexuales, transexuales, o transgénero que se oponen de igual manera a la hegemonía del patriarcado.

Detengámonos en cada uno de estos aspectos.

\section{El ciberfeminismo o tecnofeminismo}

Bajo este término se agrupan las acciones desarrolladas por los movimientos feministas en la red. Hay quienes consideran que es un movimiento que pretende colonizar el ciberespacio, quienes lo valoran como una nueva corriente feminista que rompe con el sentido más dogmático del feminismo y hasta quienes entienden que es una herramienta muy potente para dar a conocer las reivindicaciones de los movimientos feministas. "El primer éxito del ciberfeminismo se vivió en la IV Conferencia Mundial de las Mujeres de Pekín, donde un equipo de 24 mujeres de 40 países creó un espacio electrónico con información de lo que ocurría en el encuentro en 18 idiomas lo que contabilizó 100.000 visitas en la web" (Martínez y Escapa ${ }^{17}$ 2007).

De esta manera la red se ha convertido en el altavoz y punto de encuentro de mujeres de todo el mundo, creando redes de intercambio de información, conocimientos, opiniones y experiencias. Es una plataforma para la difusión de ideas, pensamientos, denuncias y modo de expresión de todas aquellas mujeres silenciadas por los medios de comunicación, porque no es noticia. Son mujeres que están informando y comunicando con perspectiva de género, mediante páginas feministas que forman parte de una gran red de una solidaridad feminista.

17. Martínez, L. y Escapa, R., Guía de formación para la participación social y política de las mujeres. Volumen II. Mérida, 2007, pp. 124. 
La red ha transformado el modo en el que nos relacionamos y comunicamos, tanto que podemos hablar de la gran aliada del feminismo en respuesta a la escasa presencia en el resto de medios de comunicación. "Internet es un excelente recurso para la colectivización y democratización del saber, para la generación de noticias alternativas, la gestión del conocimiento y el activismo social" (Martínez y Escapa, 2007). Máxime teniendo en cuenta que las redes entre mujeres son muy positivas, ya que no siempre tienen acceso a la información. Quien no tiene acceso a la información no tiene acceso al poder. Creemos necesario que las mujeres se empoderen para recuperar su espacio público en la sociedad.

Para ello, pueden beneficiarse de las ventajas que ofrecen las redes, como explican Martínez y Escapa (2007) que son: aumentan la autoestima y confianza en la ayuda, apoyo y comprensión de otras mujeres y permiten avanzar en la construcción de un modelo de sociedad diferente más equitativo y menos jerárquico, incorporando los valores que las mujeres desean aportar, redefiniendo las relaciones de poder, potenciando la comunicación interpersonal y partiendo de la "autoridad femenina", ejerciendo políticas que defiendan los intereses femeninos en todos los ámbitos sociales, lo que permitirá a las mujeres empoderarse y trabajar por una sociedad más justa.

Principalmente, algunas de las web que están haciendo ciberfeminismo son:

http://www.cyberfeminism.net/

http://www.mujeresenred.net/

http://www.genderit.org

http://ciberfeminista.org/

http://ciberfeminismo.org.ve/

http://www.lopersonalespolitico.com

http://www.rednosotrasenelmundo.org

http://www.entretodas.net

http://www.mujeresnet.info/

http://www.singenerodedudas.com

http://www.insurrectasypunto.org

http://www.e-mujeres.net/

http://www.mamametal.com

http://www.creatividadfeminista.org

http://www.donestech.net/

La mayoría en forma de blog, pero también están teniendo gran impulso a través de las redes sociales, donde numerosos colectivos de mujeres están consiguiendo difundir sus opiniones, hacer oír sus voces y crear redes sólidas de contactos, facilitando el conocimiento e intercambio. Incluso existen algunas iniciativas interesantes como http://www.takebackthetech.net/es, que pretende formar en el uso de las TIC, dominándolas para contribuir a la erradicación de la violencia contra las mujeres. 
Se trata de desafiar la concepción de que la feminidad es incompatible con los objetivos tecnológicos. "Esto supone no simplemente aceptar las innovaciones tecnológicas, sino darles forma activamente. También requiere un nuevo modelo de trabajo y vida, donde mujeres y hombres se vean igualmente representados en todos sus puestos de trabajo, incluyendo los del nivel más alto de la economía digital" (Wacjman $\left.{ }^{18}, 2008\right)$.

\section{Género e inmigración}

Hablar de inmigración nos hace caer inmediatamente en estereotipos culturales anejos a la idea de inmigrante. Este concepto previo se agrava aún más si se trata de asimilar al concepto mujer e inmediatamente se nos anticipa el concepto exclusión, con lo que hemos formado un triángulo mental que se nos fija y del que nos es difícil deshacernos.

El movimiento feminista que agrupa a varios colectivos de mujeres de culturas no occidentales está tratando de construir su propia identidad feminista para despojarlas de esos estereotipos que las estigmatizan.

En los últimos años del siglo XX se han producido varios flujos migratorios dirigidos hacia Europa como consecuencia del proceso de empobrecimiento de diversas zonas geográficas cercanas del continente africano, de diversos países del antiguo este europeo y de América del sur. La oferta de empleo para las mujeres inmigrantes se produce en los sectores de trabajo doméstico y del cuidado que son consecuencia de la incorporación mayoritaria de las mujeres europeas al mercado de trabajo y en consecuencia dejan de hacer las tareas antes realizadas por las amas de casa.

La paradoja es que aun cuando las mujeres inmigrantes se perciben como necesarias por otro lado como inmigrantes que son entran también dentro del grupo considerado una amenaza. Por ello la sociedad ahora, al contrario de otros procesos anteriores las visibiliza y se habla de ellas en los discursos políticos amplificando sus prácticas culturales y en cierto modo también considerándolas víctimas pasivas de su situación.

A ello contribuyen algunas prácticas culturales como el uso del velo o la ablación del clítoris y de supervivencia otras como la prostitución. Todo esto evidentemente las sitúa en un plano de exclusión social que las hace más vulnerables. Los estados están empezando a legislar frente a estas prácticas tomo El Consejo de Europa en la Recomendación 1325 relativa a la trata de mujeres y a la prostitución, Francia y su gobierno con sus dictámenes sobre el uso del velo en las escuelas y ahora varios municipios de Cataluña regulando el uso de prendas de tapan el rostro de las mujeres en los lugares públicos.

18. Wajcman, J., "Continuidad y cambio. Género y culturas de la tecnología y el trabajo", Revista TELOS, 74 (2008). Disponible en: [http://sociedadinformacion.fundacion.telefonica. com/telos/articuloperspectiva.asp@idarticulo=2\&rev=74.htm\#n5]. 
Carmen Gregorio ${ }^{19}$ (2002) en sus conclusiones plantea la pregunta de si cuando las políticas neoliberales propugnan la no integración de la población inmigrante no estarán ignorándose las desigualdades en el acceso a los recursos y en el ejercicio de los derechos de la ciudadanía.

Así Alicia Puleo ${ }^{20}$ es tajante cuando dice:

"un enfoque crítico de género no puede asumir un pluralismo cultural indiscriminado que, desde el relativismo ético considere a las tradiciones como horizontes cerrados que no deben ser juzgados por ningún universalismo. Tal relativismo inhabilita para dirimir conflictos entre identidades opresoras y tentativas de cambio liberador. Si el feminismo (...) se conserva fiel a su origen, su actual alineamiento con otras minorías en el proyecto multicultural no podrá ser entero y sin reservas"

El feminismo en los colectivos de mujeres inmigrantes tiene planteados varios retos que pasan por la construcción de redes de apoyo y acogida para el inicio de la vida en el nuevo país. Aquí se está haciendo patente el sentimiento de sororidad del que tantas veces se ha hablado y que les está permitiendo crear sus propios espacios de relación que les permitan superar sus miedos y conformar sus identidades y superar las dinámicas de desigualdad de sus colectivos.

Son muchas las mujeres de otras latitudes no occidentales que están trabajando por superar las desventajas en las que se encuentran y aportan desde sus intervenciones públicas y sus artículos científicos ideas que sirven al feminismo para reflexionar sobre sus posiciones y planteamientos, lo que va permitiendo poco a poco un mayor entendimiento de los presupuestos en los que se mueven (Djaouida Moualhi).

\section{La realidad transexual}

El primer problema que se nos plantea es la definición de la identidad desde la perspectiva transexual.

Existen dos perspectivas enfrentadas que intentan explicar la realidad transexual. A saber, una en la que el determinismo biológico y las diferencias orgánicas del embrión en su desarrollo, dan una explicación a la existencia de las personas trasnsexuales. Y otra de corte constructivista, que aplica la explicación sociológica y por lo tanto considera que las personas son construcciones sociales que no están supeditadas al sustrato biológico.

19. Gregorio Gil, C., Mujeres de un solo mundo: Globalización y multiculturalismo. Granada, 2002.

20. Puleo, A., "Multiculturalismo, educación multicultural y género". Tabanque, 15 (2000). 
Siguiendo a Juana Ramos (2009) ${ }^{21}$ podemos decir que desde el enfoque constructivista se han relativizado los modelos impuestos de sexo-género y se ha buscado una esencia de género escrita en algún sustrato biológico, lo que ha supuesto una especie de legitimación y de contrarrestar a quienes consideran la realidad transexual como ficciones de hembras y machos. En España se calcula que hay alrededor de 2600 transexuales lo que supone un desafío ante una realidad novedosa que implica superar esquemas mentales estereotipados y socialmente cerrados.

La pregunta que se pretende resolver es ¿Porqué una persona se siente de otro sexo-género?. La respuesta es que es una realidad y que cada persona desarrolla un sentimiento de pertenencia a cada grupo pero sin identificarse en las dicotomías establecidas y autorizadas socialmente, es decir sexo: machohembra, género: masculino-femenino. Éstos son dos modelos que tienden a desaparecer como los únicos posibles pues hoy los avances médicos que ayudan a la adaptación permiten conseguir una morfología sexual distinta.

Pero ya no todas las personas transexuales están de acuerdo con la necesidad de someterse a una cirugía moldeadora de nuevos cuerpos pues están reivindicando nuevos modelos diferentes de ser persona a los ya estereotipados hombre-mujer. Son modelos que se mantienen en los márgenes pero que día a día van adquiriendo más peso y suponen la superación del sufrimiento. A ello ha contribuido la Ley de identidad de género aprobada el 1 marzo de 2007 pues se ha conseguido facilitar, entre otras cosas el cambio de nombre y de sexo en el DNI sin necesidad de someterse a las modificaciones quirúrgicas.

Sin embargo legalmente se mantiene una impronta que relaciona a estas personas con patologías de la mente desde el momento que se sigue necesitando el certificado de disforia de sexo. Frecuentemente se confunde la transexualidad y la disforia de sexo pero son dos realidades bien distintas. La persona transexual no asume el género asignado en el nacimiento que es el que se corresponde con su sexo biológico, mientras que quien manifiesta disforia de género sufre alteraciones emocionales por ello. Pero lo que el colectivo trans denuncia es la patologización del fenómeno. Miren Larrazábal explica que estas personas deben pasar primero por la consulta del psiquiatra y después por la del psicólogo para conseguir el certificado que exige la ley.

De todas maneras la incorporación de los movimientos trans está suponiendo una renovación decisiva de la perspectiva sexo-género pues aportan argumentos que van en la línea de deconstruir la dicotomía sexual y propugnan modelos de sociedad que implican más libertad para la elección de la propia identidad sexual.

21. Ramos, J., Ponencia en Jornadas feministas "En busca de la esencia perdida; las identidades desde una perspectiva trans". Granada, 2009. 


\section{La investigación en la teoría feminista}

Una de las herramientas con las que contamos para construir y renovar la perspectiva de género y por tanto, la teoría feminista es la investigación social, que nos permite ir trazando líneas de trabajo que nos permitan dar respuesta a uno de los retos para el siglo XXI en el campo de la Ciencia, que mujeres y hombres trabajemos para obtener similar reconocimiento "en un mundo en el que ellos se consideran "la autoridad natural" (Frías Ruiz²2, 2001:173) y en él se prima el éxito de la mayoría masculina "the current system for recruitment and career development in the sciences is biased towards the success of white males, hence the lack of women and minorities in the system" (Marks²3, 2005: 790).

Cabe decir que desde sus inicios, la universidad fue un espacio de discriminación en muchos países de Europa, por ejemplo, en Reino Unido, Cambridge admitió alumnas en 1871, pero no permitió el acceso al grado de licenciatura hasta 1948, y en Oxford en 1879. Pero la discriminación de género impidió el acceso generalizado de las mujeres en igualdad con los hombres, hasta los movimientos de derechos civiles que se produjeron a partir de 1968.

De manera que en las últimas décadas, el movimiento feminista amplía su ámbito de actuación del campo político y social al académico para generar conocimiento. "Uno de los elementos que identifica el feminismo frente a otros movimientos sociales, es la producción teórica como ningún otro movimiento social en el último medio siglo" (Sendón de León ${ }^{24}$, 2003: 122). De este modo, se facilita el nacimiento y desarrollo de los Ilamados "Estudios de las Mujeres" o "Estudios de Género".

Según De Torres $^{25}$ (2000) los Estudios de Mujeres, equivalentes a los Women' Studies estadounidenses, surgidos a finales de los setenta, pretenden aplicar la perspectiva de género a cualquier campo del conocimiento, con el propósito de desenmascarar la cuestionada objetividad y neutralidad de la Ciencia y analizar la realidad teniendo en cuenta las diferencias por sexo que tienen lugar en todos los ámbitos del saber.

Por lo que no podemos dejar de afirmar junto con Isabel De Torres (2000:64) que los estudios de Mujeres "(...) están cumpliendo una interesantísima función en este final de siglo, pues han colaborado a crear una cosmovisión distinta dentro de la ciencia, en la que están presentes las mujeres, subrayando hasta qué punto el género ha distorsionado la relaciones sociales y el desarrollo vital del colectivo femenino. Puede afirmarse con toda propiedad que dichos

22. Frías Ruiz, V., Las mujeres ante la ciencia del siglo XXI. Madrid, 2001.

23. Marks Marks, A., "Sex and university system". The Journal of Clinical Investigation, 115 (2005), 790.

24. Sendón de León, $M^{a}$ V., Mujeres en la era global. Contra un patriarcado neoliberal. Barcelona, 2003.

25. De Torres, I., "El feminismo académico en España hoy". Métodos de Información, 7 (35-36) (2000), 63-67. 
Estudios representan una de las más significativas renovaciones que se han ido incorporando a la investigación en las últimas décadas".

En las universidades españolas, los Estudios de Mujeres o Estudios de Género comienzan a tener lugar, en núcleos como Madrid, Zaragoza, Valencia y Granada, con las profesoras de Enseñanza Media, pioneras españolas en las ciencias, siendo las mujeres pertenecientes a los campos de las ciencias exactas las últimas en incorporarse (Magallón ${ }^{26}$, 2004). Estos estudios por entonces sin reconocimiento ni legitimidad en los ámbitos universitarios, hasta el año 1975, fecha del Año Internacional de la Mujer y comienzo de la transición democrática.

Al realizar una breve revisión sobre los programas de doctorado, líneas de investigación y grupos de investigación que estudian el género o los estudios feministas y la educación en las universidades españolas, encontramos que en todas las universidades españolas, existen o bien programas de doctorado interuniversitario en estudios de género (universidades andaluzas y valencianas) o bien cursos de doctorado en programas de doctorado de distintas áreas de conocimiento pero que ponen el énfasis en los estudios de género o las mujeres.

En cuanto a la investigación los distintos institutos, grupos de investigación, y seminarios han generado la realización de investigaciones y trabajos académicos en torno a distintos temas como apuntaran algunos movimientos feministas.

Merecen especial atención, algunos de los que fueron pioneros en nuestro país y que propiciaron la profusión de trabajos tras ellos (De Torres, 2000):

a) Sobre el sexismo en el ámbito educativo en general, como los realizados por Subirats y Brullet $^{27}$ (1988), Garreta y Careaga ${ }^{28}$ (1987) y Alberdi ${ }^{29}$ (1999)

b) Sobre el sexismo en el lenguaje, como los de Lledó (1992) y López Valero (1995)

c) Sobre la situación de las mujeres españolas en el mercado de trabajo $M^{a}$ Ángeles Durán (1979)

De manera que desde las distintas corrientes que se agrupan bajo el movimiento feminista, encontramos diferentes temas de investigación. Mientras que las feministas liberales, se centraron en el "fracaso" de las chicas o en su bajo rendimiento en el sistema escolar y en la educación en general, ya que se elabora un discurso muy escéptico en el que se han asimilado los patrones

26. Magallón, C., "Mujeres que nos precedieron: conocerlas y reconocerlas". En Gil, A., Congreso XXV años de estudios de género. Mujeres sabias: entre la teoría y la práctica. Castellón de la Plana, 2004.

27. Subirats, M. y Brullet, C., Rosa y azul. La trasmisión de los géneros en la escuela mixta. Madrid, 1989. 1987.

28. Garreta, N y Careaga, P., Modelos masculino y femenino en los textos de EGB. Madrid,

29. Alberdi, I., "El significado del género en las Ciencias Sociales". Política y Sociedad, 32 (1999), pp. 9-21. 
masculinos de análisis, las feministas radicales se plantean la crítica y revisión de las asignaturas masculinas, los procedimientos patriarcales de la escolarización y las relaciones de poder en el aula, dando prioridad al estudio de las situaciones de opresión en la enseñanza, aulas, y otros espacios.

En la revisión de la literatura sobre la ciencia y los feminismos, encontramos posiciones ideológicas heterogéneas en relación a las aportaciones científicas intelectuales Haraway ${ }^{30}$ (1995) y Harding ${ }^{31}$ (1995), teniendo como nexo común la resistencia al discurso patriarcal dominante. La cuestión que se perfila como alternativa es el "hacer las cosas de otro modo", "consecuencia de la reinterpretación de las teorías patriarcales y de las formas nuevas de crear y enseñar los conocimientos" (Threadgold, 1999: 259). Pero, ¿cómo podremos rehacer el medio académico desde su interior, sin perder credibilidad en dicho medio?. Quizás sea posible, parafraseando a Bourdieu, construir un tipo de habitus ya diferente o reconstruir un habitus ya hecho (Threadgold ${ }^{32}$, 1999).

Diana Sartori ${ }^{33}$ (1998) recapitula el estado de la cuestión de la investigación femenina que hace referencia a la ciencia, diciendo que no es posible la agrupación debido a la profusión de trabajos en las últimas décadas, aunque cita a autoras que establecen algunas clasificaciones al respecto.

Massó $^{34}$ (2004) recoge siguiendo a Pérez Sedeño (1997), las áreas en las que se agrupan los estudios realizados desde los feminismos en el campo de la ciencia:

- Área histórico-sociológica, donde se estudia el acceso e incorporación de las mujeres a los campos de la ciencia y la tecnología

- Área pedagógica, que trabajan con el propósito de aumentar la presencia de las mujeres en las ciencias, desde una educación libre de sesgos sexistas

- Área epistemológica, donde tienen lugar las disputas teóricas más encontradas

Por tanto, el eje que articula las críticas feministas a la ciencia tradicional es el sesgo androcéntrico de la investigación. Las prácticas científicas convencionales contienen un sesgo androcéntrico evidente, tanto en los conceptos y las categorías que utilizan en la indagación científica, como en los marcos teóricos en los que se inscriben. Por un lado, provoca discriminación en las mujeres para el acceso a la ciencia, y por otro, es la causa de la "mala ciencia" y/o resultados "sesgados".

30. Haraway, D., Ciencia, Cyborgs y mujeres. La reinvención de la naturaleza. Madrid, 1995.

31. Harding, S., Ciencia y feminismo. Madrid, 1996.

32. Threadgold, T., "La vida cotidiana en el mundo académico: Feminismos postmodernos, seducciones genéricas, reescritura y hacerse escuchar". En Luke, C. (comp.), Feminismos y pedagogías de la vida cotidiana. Madrid, 1999. (Traducción castellano).

33. Sartori, D., "La autoridad en cuestión". En Colectivo Hipatia, Autoridad científica, autoridad femenina. Madrid, 1998.

34. Massó Massó, E., "Género y ciencia. Una relación fructífera" [en línea]. Gazeta de Antropología, 20 (2004). <http://www.ugr.es/pwlac/G20_06Ester_Masso_Guijarro.html>. 
La epistemología feminista aboga por la pluralidad de discursos y prácticas en el uso de la ciencia, por el reconocimiento de aquellas ideas, teorías, métodos, técnicas y visiones poco valoradas hasta ahora, que supongan un enriquecimiento de la metodología científica y no la uniformidad científica, discurso tradicional de la ciencia, puesto que la ciencia no ha sido neutral, ni objetiva ni formal con las mujeres, como ya han puesto de manifiesto varias autoras como Claramunt ${ }^{35}$ et. al., (2002), Santesmases ${ }^{36}$ (2000), García de León ${ }^{37}$ (2002), Flecha García ${ }^{38}$ (2003), Pérez Sedeño ${ }^{39}$ et. al. (2003) y Gutiérrez Esteban (2004). Fueron los análisis feministas quienes evidenciaron la ocultación o invisibilización que se había hecho de la participación de las mujeres, ya que la ciencia está hecha y escrita por y para los hombres. Podemos decir que no ha habido muchísimas mujeres en las ciencias, porque su entrada en la educación reglada fue posterior; sin embargo, ha habido más de las que la historia ha presentado (Pérez Sedeño ${ }^{40}$, 2004).

Por ello surge la necesidad de crear una epistemología, una teoría y una metodología propia, distinta, que reelabore el pensamiento y el conocimiento científico desde una nueva perspectiva, la perspectiva de género. Pues la ciencia ha sido tradicionalmente patriarcal y androcéntrica excluyendo a las mujeres y los temas que interesaban y/o tenían en cuenta a las mujeres.

Aquí entraría en juego la dialéctica del feminismo dialógico, para que la ciencia fuese imparcial debería oír todas las voces que la componen, de manera inclusiva e igualadora (Puigvert, 2001, Gutiérrez Esteban¹, 2005 y Arrufat Gallardo, 2004).

\section{Ideas finales}

Descubrimos que en cualquier época, tanto desde las primeras sufragistas como hasta las seguidoras del cyberfeminismo, se han dado cuenta de que en aquellos tiempos no fue suficiente, ni lo es hoy en día, con cambiar leyes y denunciar hechos injustos muestra de la desigualdad por razón de género. Las acciones son las que hacen que cambien las actitudes y comportamientos. Y

35. Claramunt, R. Ma , Portela Peñas, I. y Claramunt, T., Las mujeres en las Ciencias Experimentales. Madrid, 2002.

36. Santesmases, M. J., Mujeres científicas en España (1940-1970). Madrid, 2000.

37. García de León, M. A., Herederas y heridas: sobre las élites profesionales femeninas. Madrid, 2002.

38. Flecha García, C., "Educación y Género. Una crítica a la Teoría del Capital Humano". Curso de Postgrado Nuevas Perspectivas para el Empleo de las Mujeres. Sevilla, 2003. Documento inédito.

39. Pérez Sedeño, E. (dir.), "La situación de las mujeres en el sistema educativo de ciencia y tecnología y su contexto internacional" [en línea]. Madrid, 2003. http://www.tecnociencia. es/fecyt/uploads/mujerescienciaytecnologia_63.pdf [consulta 7/7/2004, 11:22].

40. Pérez Sedeño, E., "A la sombra del varón: hijas, esposas, hermanas". Mesa redonda "Mujeres sabias en Ciencia y Tecnología". Congreso XXV años de estudios de género. Mujeres sabias, entre la teoría y la práctica. Castellón 29 septiembre y 1 octubre de 2004.

41. Gutiérrez Esteban, P., "As mulheres na ciencia. Perda de capital humano e tecto de cristal". En E. Figueira y L. Rainha (coords.), Qualificação e Género: O papel das competênciaschave. Èvora, 2005, pp. 71-89. 
son gracias a éstas y a ellas, desde los inicios de los movimientos feministas, las que han hecho posible configurar las teorías feministas, unos modos de pensar, sentir y actuar, que salvando las diferencias entre unas y otras corrientes, han permitido al resto de las mujeres apoyarse en una teoría que les permitiese analizar y denunciar su situación.

Esto ilustra la situación de mujeres y hombres en un mundo cada vez más tecnificado y con mayores índices de progreso, que según los baremos que se utilizan para medir estos avances, sigue presentando las mismas o mayores desigualdades entre las personas que hace un par de siglos o más, apareciendo incluso nuevas formas de desigualdad como la brecha digital (Castaño, 2005 ${ }^{42}$ ). Lo que demuestra que a pesar de la lucha y los esfuerzos de mujeres y hombres por cambiar esta realidad desigual, no ha sido suficiente para cambiar las estructuras sociales patriarcales y androcéntricas que determinan su funcionamiento.

"Queda pendiente en este sentido el trabajo de sistematizar y organizar un discurso propio feminista que suponga un avance en el conocimiento científico educativo actual, así como la organización de una agenda crítica feminista en el campo educativo" Colás ${ }^{43}$ (2003:10-11).

Finalmente estamos de acuerdo con la reivindicación general de todas las mujeres de la necesidad de conseguir la sororidad, concepto acuñado por Lagarde $^{44}$ (1996) y que implica la solidaridad entre nosotras.

"La libertad femenina está en esa tesitura y búsqueda de tensión siempre en el sentido libre -la interpretación de la experiencia femenina libre, que la hay- y el orden sociosimbólico dominante que la mantiene inaudita. La posibilidad de la libertad se abre, por propia experiencia y las de otras, cuando sabemos sustraernos a lo que viene dado como objetivado y universal con un sentido pensado y pensable del vivir y de las relaciones abstrayendo, repito, lo que cada una y uno es, abstrayendo así lo que excede que pide ser pensado como nuevo y no repetido, como es el sentido libre de la diferencia sexual del ser mujer y también del ser hombre" (Arnaus ${ }^{45}$, 2010: 161).

Pensamos que si las mujeres consiguen resignificar su subjetividad renunciando al rol de mujer victimizada por el sistema patriarcal, en el deseo de hacer realidad la construcción de espacios de relación que nos permitan tejer complicidades y urdir confianzas. Viviremos nuestra esencia de ser mujer como seres inteligentes, capaces y libres.

42. Castaño, C., Las mujeres y las tecnologías de la información. Internet y la trama de nuestra vida. Madrid, 2005.

43. Colás, P., "Investigación educativa y crítica feminista" [en línea]. Ágora digital, 6 (2003), pp. 1-20. <http://www.agoradigital.com> [consulta: 07/07/03, 21:07].

44. Lagarde, M., Género y feminismo: desarrollo humano y democracia. Madrid, 1996.

45. Arnaus, R., "El sentido libre de la diferencia sexual en la investigación educativa". En Contreras, J. y Pérez de Lara, N. (comps.), Investigar la experiencia educativa. Madrid, 2010. 